\title{
REGIONAL SAR IMAGE SEGMENTATION BASED ON FUZZY CLUSTERING WITH GAMMA MIXTURE MODEL
}

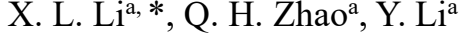 \\ ${ }^{a}$ Institute for Remote Sensing Science and Application, School of Geomatics, Liaoning Technical University, \\ Fuxin, Liaoning 123000, China - (lixiaolilntu, zqhlby, lntuliyu)@163.com
}

\section{Commission I, WG I/3}

KEY WORDS: Synthetic Aperture Radar (SAR), Image segmentation, Gamma mixture model (GaMM), Fuzzy clustering, Markov Random Field (MRF)

\begin{abstract}
:
Most of stochastic based fuzzy clustering algorithms are pixel-based, which can not effectively overcome the inherent speckle noise in SAR images. In order to deal with the problem, a regional SAR image segmentation algorithm based on fuzzy clustering with Gamma mixture model is proposed in this paper. First, initialize some generating points randomly on the image, the image domain is divided into many sub-regions using Voronoi tessellation technique. Each sub-region is regarded as a homogeneous area in which the pixels share the same cluster label. Then, assume the probability of the pixel to be a Gamma mixture model with the parameters respecting to the cluster which the pixel belongs to. The negative logarithm of the probability represents the dissimilarity measure between the pixel and the cluster. The regional dissimilarity measure of one sub-region is defined as the sum of the measures of pixels in the region. Furthermore, the Markov Random Field (MRF) model is extended from pixels level to Voronoi sub-regions, and then the regional objective function is established under the framework of fuzzy clustering. The optimal segmentation results can be obtained by the solution of model parameters and generating points. Finally, the effectiveness of the proposed algorithm can be proved by the qualitative and quantitative analysis from the segmentation results of the simulated and real SAR images.
\end{abstract}

\section{INTRODUCTION}

Synthetic Aperture Radar (SAR) (Bombrun et al., 2011) as an important technique of remote sensing is widely used in many fields. SAR image segmentation plays a key role in image interpretation (Dass et al., 2012). However, there is much speckle noise in SAR images due to the special imaging mechanism which bring great difficulty in SAR image segmentation (Intajag and Chitwong, 2016).

Recently, fuzzy clustering algorithm shows the strong advantage in image segmentation (Tehrani and Ibrahim, 2014; Sajith and Hariharan, 2015), the fuzzy membership can deal with the problem of uncertainty. However, the similarity measure based on Euclidean distance is extremely sensitive to noise (Liu et al., 2009). For complex images, the stochastic model is regard as the more effective way. The traditional stochastic algorithms often assume that the pixels intensities in one cluster obey Gaussian distribution (Hou et al., 2010; Nguyen and $\mathrm{Wu}, 2013)$. Chatzis and Varvarigou (2008) proposed hidden Markov random field FCM (HMRF-FCM), it defines the dissimilarity measure by Gaussian distribution in fuzzy clusters, and the prior probability is defined by HMRF to consider the effects of neighbor pixels. The algorithm has been successfully applied to many image segmentation fields. Furthermore, considering the speckle noise in SAR image, Gamma distribution is often adopted to depict the stochastic characteristic of pixels (Lopes et al., 1990). Although Dong et al. (1998) have proved that Gamma distribution is more applicable for SAR image than Gaussian distribution, the histogram distribution in one cluster usually tends to have multimodal characteristics which are difficult to be fitted by one gamma distribution (Peng et al., 2013). Kayabol and Gunsel (2013) propose a Bayesian Gamma mixture model based unsupervised classification of SAR images, and the benefited are validated effectively. However, the algorithms talked above are all pixels-based, which still can not overcome the speckle noise well in SAR image segmentation.

In order to solve the problems, a regional SAR image segmentation algorithm based on fuzzy clustering with Gamma mixture model (VT-GaMM-FCM) is proposed. First, the image domain is divided into many sub-regions by some generating points using Voronoi tessellation technique (Dryden et al., 2006; Nithyakalyani and Kumar, 2014). The sub-regions corresponding to each generating point are constituted of the pixels with the minimum Euclidean distance to the point. Based on the Voroonoi polygons, assume that the pixels within the polygon follows GaMM, and the dissimilarity measure of the polygon is the sum of the measure of pixels. In addition, the prior probability which is the weight of GaMM is introduced on polygon label field under Markov random field (MRF) theory to describe the effects of neighbor polygons label. Furthermore, the prior probability is also used in the regularization term to control the clustering scale. After establishing objective function extended from HMRF-FCM, the 
solution of the segmentation model includes solving the model parameters and updating generating point. For the model parameters solution, like membership degree and scale parameter, they can be directly obtained by the derivation of object function. For the move of updating generating point, a generating point is randomly selected to move into the candidate points existed in the sub-region, and the tessellation is updated with the shape changing of the sub-region. Then, A new segmentation result under the current tessellation is obtained. The acceptance of the moving is the decrease of the objective function. After several iterations, the optimal segmentation results can be found.

\section{DESCRIPTION OF THE PROPOSED ALGORITHM}

\subsection{Image expression}

Assume that $z=\left\{z_{i}\left(x_{i}, y_{i}\right), i=1, \ldots, n\right\}$ is a SAR image in image domain $\boldsymbol{\Omega}$, where $i$ is the index of pixels, $z_{i}$ and $\left(x_{i}, y_{i}\right)$ are the intensity and position of pixel $i$, respectively, $n$ is the number of pixels. $z$ can be regarded as the concrete realization of the characteristic field $\boldsymbol{Z}=\left\{Z_{i}, i=1, \ldots, n\right\}, \boldsymbol{Z}$ is a random field about intensities.

The image domain $\boldsymbol{\Omega}$ is divided into many sub-regions by generating points using Voronoi tessellation, $\boldsymbol{P}=\left\{P_{j}, j=1, \ldots, m\right\}$ where $j$ is the index of polygons, $P_{j}$ represents polygon $j, m$ is the number of polygons. The generating points $\boldsymbol{G}=\left\{\left(a_{j}, b_{j}\right):\left(a_{j}\right.\right.$, $\left.\left.b_{j}\right) \in \boldsymbol{\Omega}\right\}$, where $\left(a_{j}, b_{j}\right)$ is the position of generating point $j$, and the polygon $P_{j}$ is consist of the pixels with the minimum Euclidean distance from $\left(a_{j}, b_{j}\right)$.

Assume that the image has $c$ clusters, the label field $\boldsymbol{L}$ can be express as $\boldsymbol{L}=\left\{L_{i}, i=1, \ldots, n\right\}$ or $\left\{L_{j}, j=1, \ldots, m\right\}, L_{i}$ is described on pixel, $L_{j}$ is described on polygon, and $\boldsymbol{l}=\left\{l_{i}, i=1, \ldots, n\right\}=\{l$, , $j=1, \ldots, m\}$ are the concrete realization of the label field, and $\boldsymbol{l} \in\{1, \ldots, c\}$, If and only if $z_{i} \in P_{j}, l_{i}=l_{j}$.

\subsection{Segmentation model}

Combining fuzzy cluster segmentation algorithms, the membership matrix is used to describe the relationship between polygons and clustering, $\boldsymbol{U}=\left[u_{j k}\right]_{m \times c}$, where $k$ is the index of clusters, and $\boldsymbol{U}$ satisfied $0 \leq u_{j k} \leq 1 ; \sum_{k=1}^{c} u_{j k}=1, \forall j=1, \ldots, m$; $0<\sum_{j=1}^{m} u_{j k}<m, \forall k=1, \ldots, c$.

In order to extend the segmentation model from pixels to Voronoi polygons, The objective function of the proposed algorithm is defined as,

$$
J=\sum_{j=1}^{m} \sum_{k=1}^{c} u_{j k} D_{j k}+\lambda \sum_{j=1}^{m} \sum_{k=1}^{c} N_{j} u_{j k} \log \frac{u_{j k}}{\pi_{j k}}
$$

Where $\lambda$ is fuzzy factor, $N_{j}=\#\left\{z_{i},\left(x_{i}, y_{i}\right) \in P_{j}\right\}$ is the number of pixels within polygon $j, \pi_{j k}$ is prior probability about polygon $j$ belonging to cluster $k, D_{j k}$ is the dissimilarity measure between polygons and the cluster center, which is defined as the sum of all pixels within polygon $j, \boldsymbol{D}=\left[D_{j k}\right]_{m \times c}$.

$$
D_{j k}=\sum_{\left(x_{i}, y_{i}\right) \in P_{j}} d_{i k}
$$

where $d_{i k}$ is the dissimilarity measure between pixels and the cluster center. Based on statistic model, the dissimilarity is usually defined by the negative logarithm of probability distribution. In order to describe the multimodal characteristic, $d_{i k}$ is described with GaMM,

$$
d_{i k}=-\log \pi_{j k} p\left(z_{i} \mid \boldsymbol{\theta}_{k}\right)
$$

where $z_{i} \in P_{j}, \boldsymbol{\theta}_{k}=\left(\alpha_{k}, \beta_{k}\right), \alpha_{k}$ and $\beta_{k}$ are the shape and scale parameters of Gamma distribution, respectively, $p\left(z_{i} \mid \boldsymbol{\theta}_{k}\right)$ is the probability density function of Gamma distribution. In this paper, the shape parameter $\alpha_{k}$ is equal to the number of looks, thus, $p\left(z_{i} \mid \boldsymbol{\theta}_{k}\right)$ can be written as,

$$
p\left(z_{i} \mid \beta_{k}\right)=\frac{z_{i}^{\alpha-1}}{\Gamma(\alpha) \beta_{k}^{\alpha}} \exp \left(-\frac{z_{i}}{\beta_{k}}\right)
$$

In addition, in order to consider the effects of neighbor polygons, the spatial constraint is added to the prior probability. Assume that $\partial_{j}$ is the neighborhood set of polygon $j, \partial_{j}=\left\{P_{j}\right.$, $\left.j^{\prime} \neq j\right\}$ and satisfied $j \in \partial_{j}, j^{\prime} \in \partial_{j}$. Based on the Markov random field (MRF) theory, the prior probability is defined as,

$$
\pi_{j k}=p\left(l_{j}=k \mid l_{j^{\prime}}, j^{\prime} \in \partial_{j}\right)=\frac{\exp \left(\eta \sum_{j^{\prime} \in \partial_{j}} \delta\left(l_{j}=k, l_{j^{\prime}}\right)\right)}{\sum_{k^{\prime}=1}^{c} \exp \left(\eta \sum_{j^{\prime} \in \partial_{j}} \delta\left(k^{\prime}, l_{j^{\prime}}\right)\right)}(5)
$$

Where $\eta$ is the intensity of neighborhood effect, $\delta$ is an energy function, $\delta(x, y)=1$, if and only if $x=y$.

\subsection{Parameter estimation}

The parameter estimation includes two aspects, one is the model parameters about $u_{j k}$ and $\beta_{k}$, which can be obtained directly by the derivative of the objective function. Another is the solution of generating points $\boldsymbol{G}$, which need moving operation to find the best Voronoi tessellation.

2.3.1 Solution model parameters: Because $u_{j k}$ has constraint condition, thus, the Lagrange equation need to be built firstly, let it equal to 0 ,

$$
\frac{\partial}{\partial u_{j k}}\left[J-\sum_{j=1}^{m} \xi_{j}\left(\sum_{k^{\prime}=1}^{c} u_{j k^{\prime}}-1\right)\right]=0
$$

Then the membership $u_{j k}$ is,

$$
u_{j k}=\frac{\pi_{j k} \exp \left(-\frac{1}{\lambda N_{j}} D_{j k}\right)}{\sum_{k^{\prime}=1}^{c} \pi_{j k^{\prime}} \exp \left(-\frac{1}{\lambda N_{j}} D_{j k^{\prime}}\right)}
$$

For scale parameter $\beta_{k}$, it can be obtained by making the derivative equal to 0 , 


$$
\beta_{k}=\frac{\sum_{j=1}^{m}\left(u_{j k} \sum_{\left(x_{i}, y_{i}\right) \in P_{j}} z_{i}\right)}{\alpha \sum_{j=1}^{m} N_{j} u_{j k}}
$$

2.3.2 Updating generating points: In order to complete the optimal segmentation, the Voronoi tessellation is changed by moving generating points under the condition of minimizing objective function.

Assume that $t$ is the current iteration, the generating points set $\boldsymbol{G}^{(t)}=\left\{\left(a_{1}{ }^{(t)}, b_{1}{ }^{(t)}\right), \ldots,\left(a_{j}^{(t)}, b_{j}^{(t)}\right), \ldots,\left(a_{m}{ }^{(t)}, b_{m}{ }^{(t)}\right)\right\}$. Randomly selected a polygon $j$ and the corresponding generating point $\left(a_{j}^{(t)}, b_{j}^{(t)}\right)$. In polygon $j$, randomly selected a candidate generation point $\left(a_{j}^{*}, b_{j}^{*}\right),\left(a_{j}{ }^{*}, b_{j}^{*}\right) \in P_{j}$, and $\left(a_{j}^{*}, b_{j}^{*}\right) \neq\left(a_{j}{ }^{(t)}, b_{j}{ }^{(t)}\right)$. Moving $\left(a_{j}^{(t)}, b_{j}^{(t)}\right)$ to $\left(a_{j}^{*}, b_{j}^{*}\right)$, then the new generating points set is $\boldsymbol{G}^{*}=\left\{\left(a_{1}{ }^{(t)}, b_{1}{ }^{(t)}\right), \ldots,\left(a_{j}^{*}, b_{j}^{*}\right), \ldots,\left(a_{m}{ }^{(t)}, b_{m}{ }^{(t)}\right)\right\}$. According to the new generating points, the image is segmented again. The objective function $J^{*}$ is recalculated by new model parameter $u_{j k}{ }^{*}$ and $\beta_{k}{ }^{*}$. Comparing with $J^{*}$ and $J^{(t)}$, if $J^{*}<J^{(t)}$, the moving is accepted, and updating the model parameters $u_{j k}{ }^{(t+1)}=u_{j k}{ }^{*}, \beta_{k}{ }^{(t+1)}=\beta_{k}{ }^{*}$, and $\boldsymbol{G}^{(t+1)}=\boldsymbol{G}^{*}$, otherwise, refused the moving and the parameters and generating points are remained unchanged.

\section{EXPERIMENTAL RESULTS AND DISCUSSION}

In order to evaluate the proposed algorithm, the experimental is designed on the simulated and real SAR images with two comparing algorithms and the proposed algorithm. For comparing algorithms, one is Gamma-FCM which is defined with Gamma distribution, another is GaMM-FCM which is defined with GaMM, both of them are pixel-based.

\subsection{Simulated image}

Figure $1(b)$ is the simulated image generated by template with four homogeneous regions shown in Figure 1 (a). Figure 1 (b) is used to simulate multi-look SAR image and the parameters are listed in Table 1. Figure 1 (b) is obtained by four random numbers with the parameters.

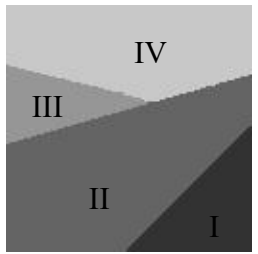

(a) Template

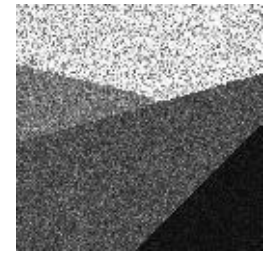

(b) Simulated
Figure 1. Template and simulated image.

\begin{tabular}{ccccc}
\hline \multirow{2}{*}{ Parameters } & \multicolumn{4}{c}{ Homogeneous regions } \\
\cline { 2 - 5 } & I & II & III & IV \\
\hline$\alpha$ & 4 & 4 & 4 & 4 \\
$\beta$ & 5 & 20 & 30 & 65 \\
\hline
\end{tabular}

Table 1. Shape and scale parameters of Gamma distribution

Figure 2 are the segmentation results with the proposed algorithm. Where Figure 2 (a1)-(c1) are the Voronoi tessellation with initial state, 500 iterations and the final result, Figure 2 (a2)-(c2) are the corresponding segmentation results. Figure 2 effectively prove the fitting ability of the homogeneous regions boundary using Voronoi polygons, and the segmentation result is more and more accurate with the increase of iterations.

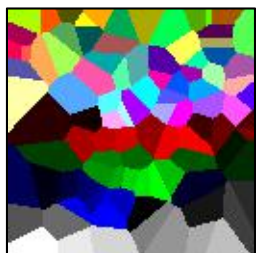

(a1)

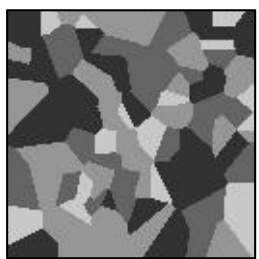

(a2)

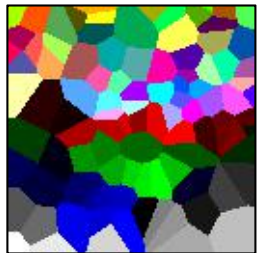

(b1)

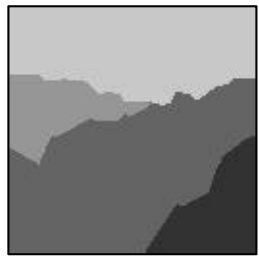

(b2)

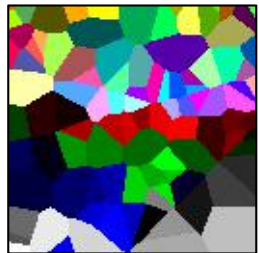

(c1)

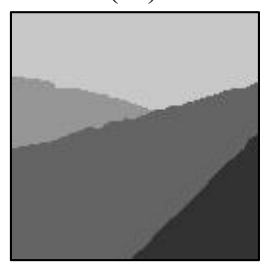

(c2)
Figure 2. Segmentation results with initial state, 500 iterations and final result.

In order to qualitatively analyze the segmentation results, the segmentation results with comparing algorithms are shown in Figure 3. Where Figure 3 (a1)-(c1) are the segmentation results with Gamma-FCM, GaMM-FCM and the proposed algorithm, Figure 3 (a2)-(c2) are the outline added images, respectively. Figure 3 show that there are many error points in the comparing algorithms, and the segmentation results with the proposed algorithm are much better than comparing algorithms.

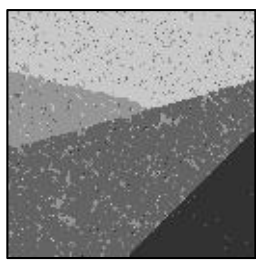

(a1)

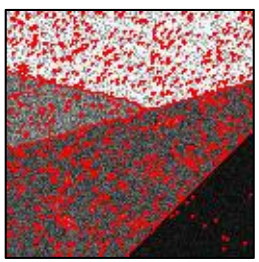

(a2)

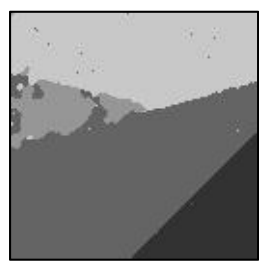

(b1)

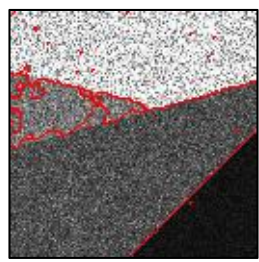

(b2)

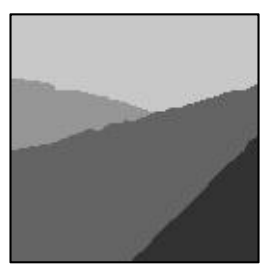

(c1)

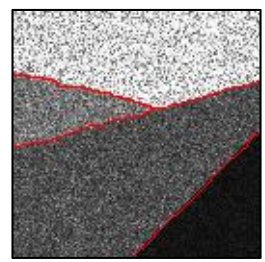

(c2)
Figure 3. Segmentation results and outline added images.

In order to quantitatively analyze the segmentation results, taking Figure 1 (a) as the standard segmentation, and calculating the confusion matrix, then the user's, product's, overall accuracy and Kappa value are listed in Table 2. It shows that the segmentation accuracy and kappa value of the proposed algorithm is $95 \%$ and 0.98 , respectively, which is much higher than the comparing algorithms.

\begin{tabular}{|c|c|c|c|c|c|}
\hline \multirow{2}{*}{ Algorithms } & \multirow{2}{*}{$\begin{array}{c}\text { Accuracy } \\
(\%)\end{array}$} & \multicolumn{4}{|c|}{ Homogeneous regions } \\
\hline & & I & II & III & IV \\
\hline \multirow{2}{*}{$\begin{array}{c}\text { Gamma } \\
\text { FCM }\end{array}$} & User's & 99.41 & 89.48 & 93.08 & 92.15 \\
\hline & Product's & 98.70 & 97.79 & 58.74 & 100 \\
\hline
\end{tabular}




\begin{tabular}{cccccc} 
GaMM & User's & 99.91 & 99.92 & 70.23 & 99.27 \\
FCM & Product's & 100 & 94.39 & 97.72 & 98.84 \\
\multicolumn{5}{c}{ overall $(\%)=96.83 ; \mathrm{Kappa}=0.95$} \\
VT-GaMM & User's & 98.46 & 99.71 & 95.53 & 99.74 \\
FCM & Product's & 99.95 & 98.89 & 98.70 & 99.29 \\
& \multicolumn{5}{c}{ overall $(\%)=99.15 ; \mathrm{Kappa}=0.99$} \\
\hline
\end{tabular}

Table 2. Comparison of accuracies and kappa value

\subsection{Real SAR image}

To demonstrate the universal applicability of the proposed algorithm further, Figure 4 with three real SAR images are used to test. All of them come from RADARSAT-I/II with $128 \times 128$ pixels.

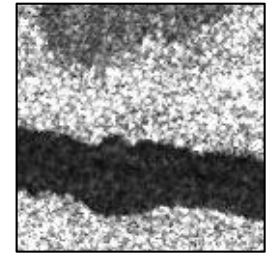

(a)

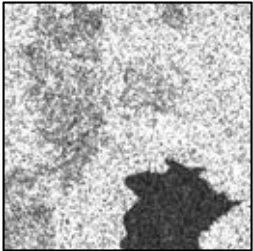

(b)

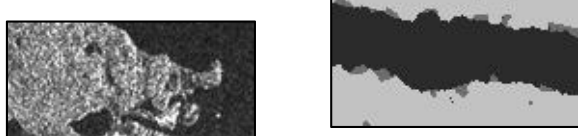

(a1)

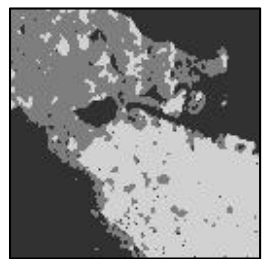

(c1)

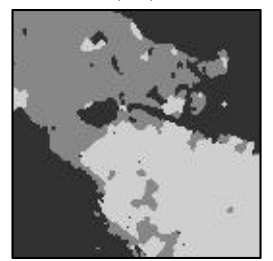

(c1) (c)

Figure 4. Real SAR images.

Figure 5 are the segmentation results with the proposed algorithm, where Figure 5 (a1)-(c1) are the Voronoi tessellation results, Figure 5 (a2)-(c2) are the corresponding segmentation results, Figure 5 (a3)-(c3) are the outline added images. Figure 5 show that the proposed algorithm is applicable to the real SAR images, additionally, it can effectively overcome the effects of noises and the fitting of boundary is accurate.

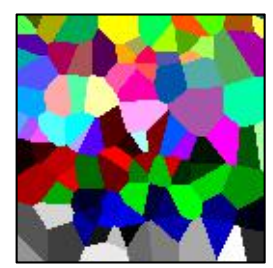

(a1)

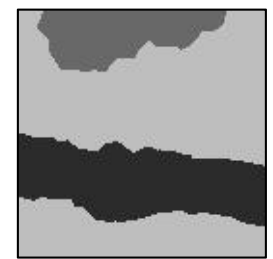

(a2)

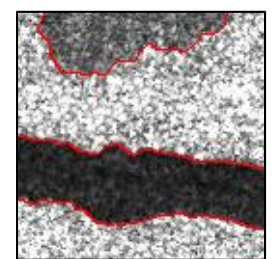

(a3)

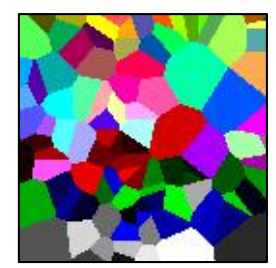

(b1)

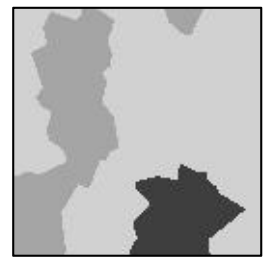

(b2)

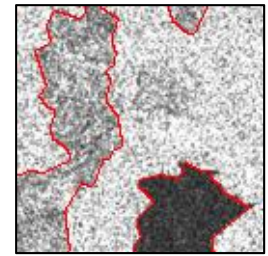

(b3)

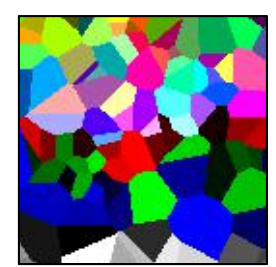

(c1)

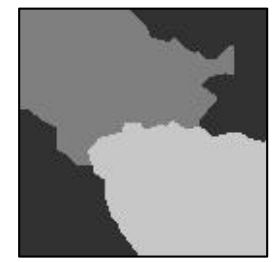

(c2)

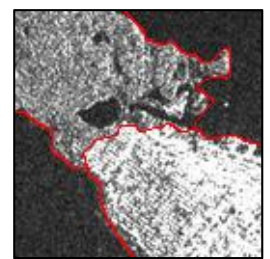

(c3)
Figure 5. Segmentationo results with the proposed algorithm for real SAR images.

Figure 6 are the segmentation results with comparing algorithms, where Figure 6 (a1)-(c1) are Gamma-FCM, Figure

6 (a2)-(c2) are GaMM-FCM. Figure 6 show that GaMM-FCM is better than Gamma-FCM, but it still not as good as the

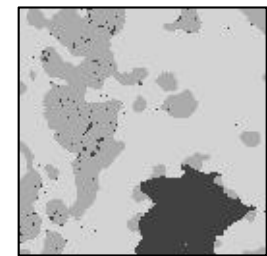

(b1)

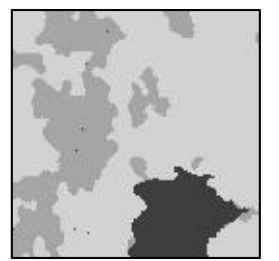

(b1)
Figure 6. Segmentation results with comparing algorithms for

real SAR images.

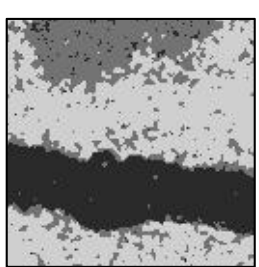

(a1)

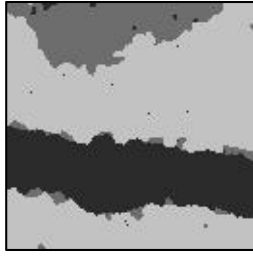

\section{CONCLUSION}

In this paper, a regional SAR image segmentation algorithm based on fuzzy clustering with Gamma mixture model is proposed. Combing Voronoi tessellation, the Gamma mixture model is used to define the dissimilarity measure in the polygon. With the help of prior probability as the weight defined by MRF, the proposed algorithm can not only overcome the effect of noise well, but also accurately describe the multimodal characteristics distribution of the image. The experiments with comparing algorithms and the proposed algorithm show that GaMM is better than Gamma distribution and VT-GaMM is better than GaMM in fuzzy clustering for SAR image segmentation. In the future, the aim for the study is to segment SAR image with the changing shape parameter.

\section{REFERENCES}

Bombrun, L., Vasile, G., Gay, M., Totir, F., 2011. Hierarchical segmentation of polarimetric SAR images using heterogeneous clutter models. IEEE Transactions on Geoscience and Remote Sensing, 49(2), pp. 726-737.

Chatzis, S., Varvarigou, T., 2008. A fuzzy clustering approach toward hidden Markov random field models for enhanced spatially constrained image segmentation. IEEE Transactions on Fuzzy Systems, 16(5), pp. 1351-1361.

Dass, R., Priyanka, Devi, S., 2012. Image segmentation techniques. International Journal of Electronics \& Communication Technology, 3(1), pp. 2230-7109.

Dong, Y., Forester, B., Milne, A., 1998. Evaluation of radar image segmentation by Markov random field model with Gaussian distribution and Gamma distribution. In: 1998 IEEE International Geoscience and Remote Sensing, Seattle, USA, pp. 1617-1619.

Dryden, I. L., Famoosh, R., Taylor, C. C., 2006. Image segmentation using Voronoi polygons and MCMC, with application to muscle fiber images. Journal of Applied Statistics, 33(6), pp. 609-622. 
Hou, Y., Sun, X., Lun, X., Lan, J., 2010. Gaussian mixture model segmentation algorithm for remote sensing image. In: 2010 International Conference on Machine Vision and Humanmachine Interface, pp. 275-278.

Intajag, S., Chitwong, S., 2016. Speckle noise estimation with generalized Gamma distribution. In: International Joint Conference, Busan, Korea, pp. 201610 18-21.

Kayabol, K., Gunsel, B., 2013. SAR image classification with normalization Gama process mixtures. In: 2013 IEEE International Conference on Image Processing Image Processing, Australia, pp. 320-324.

Liu, H., Jeng, B., Yih, J., Yu, Y., 2009. Fuzzy c-means algorithm based on standard Mahalanobis distances. In: 2009 International Symposium on Information Processing, Huangshan, China, pp. 422-427.

Lopes, A., Laur, H., Nezry, E., 1990. Statistical distribution and texture in multilook and complex SAR Images. In: International Geoscience and Remote Sensing Symposium, pp. $2427-2430$.

Nguyen, T. M., Wu, Q. M., 2013. Fast and robust spatially constrained Gaussian mixture model for image segmentation. IEEE Transactions on Circuits and Systems for Video Technology, 23(4), pp. 621-635.

Nithyakalyani, S., Kumar, S. S., 2014. Voronoi fuzzy clustering approach for data processing in WSN. International Journal of Computational Intelligence Systems, 7(1), pp. 105-113.

Peng, Y., Chen, J., Xu, X., Pu, F., 2013. SAR Images statistical modeling and classification based on the mixture of AlphaStable distributions. Remote Sensing, 5(5), pp. 2145-2163.

Sajith, A. G., Hariharan, S., 2015. Spatial fuzzy C-means clustering based segmentation on CT images. In: $20152^{\text {nd }}$ International Conference on Electronics and Communication Systems, Coimbatore, pp. 2015: 26-27.

Tehrani, I. O., Ibrahim, S., 2014. An enhanced fuzzy c-means medical segmentation algorithm. In: 2014 International Symposium on Biometrics and Security Technologies (ISBAST), Kuala, Lumpur, pp. 2014: 285-289.

Revised May 2017 